\title{
Incidence and Significance of Biliary Stricture in Chronic Pancreatitis Patients Undergoing Extracorporeal Shock Wave Lithotripsy for Obstructing Pancreatic Duct Stones
}

\author{
Jong Jin Hyun ${ }^{1,2}$, Shayan S. Irani², Andrew S. Ross², Michael C. Larsen², Michael Gluck², and Richard A. Kozarek² \\ ${ }^{1}$ Department of Internal Medicine, Korea University Ansan Hospital, Ansan, Korea, and ${ }^{2}$ Digestive Disease Institute, Virginia Mason \\ Medical Center, Seattle, WA, USA
}

\section{Article Info}

Received November 1, 2019

Revised January 4, 2020

Accepted January 4, 2020

Published online May 13, 2020

\section{Corresponding Author}

Richard A. Kozarek

ORCID https://orcid.org/0000-0002-9745-7538

E-mail Richard.Kozarek@virginiamason.org

\begin{abstract}
Background/Aims: This study assessed the significance of biliary stricture in symptomatic chronic pancreatitis patients requiring extracorporeal shock wave lithotripsy (ESWL) and endoscopic retrograde cholangiopancreatography (ERCP) to remove obstructing pancreatic calculi.

Methods: A total of 97 patients underwent ESWL followed by ERCP to remove pancreatic calculi between October 2014 and October 2017 at Virginia Mason Medical Center. Significant biliary stricture (SBS) was defined as a stricture with upstream dilation on computed tomography scan or magnetic resonance cholangiopancreatography scans accompanied by cholestasis and/or cholangitis. SBS was initially managed by either a plastic stent or fully covered self-expandable metallic stent (fcSEMS). If the stricture did not resolve, the stent was replaced with either multiple plastic stents or another fcSEMS. Data were collected by retrospectively reviewing the medical records.

Results: Biliary strictures were noted in approximately one-third of patients $(34 / 97,35 \%)$ undergoing ESWL for pancreatic calculi. Approximately one-third of the biliary strictures $(11 / 34,32 \%)$ were SBS. Pseudocysts were more frequently found in those with SBS ( $36 \%$ vs $8 \%, p=0.02$ ), and all pseudocysts in the SBS group were located in the pancreatic head. The initial stricture resolution rates with fcSEMSs and plastic prostheses were $75 \%$ and $29 \%$, respectively. The overall success rate for stricture resolution was $73 \%(8 / 11)$, and the recurrence rate after initial stricture resolution was $25 \%(2 / 8)$.

Conclusions: Although periductal fibrosis is the main mechanism underlying biliary stricture development in chronic pancreatitis, inflammation induced by obstructing pancreatic calculi, including pseudocysts, is an important contributing factor to SBS formation during the acute phase. (Gut Liver 2021;15:128-134)
\end{abstract}

Key Words: Chronic pancreatitis; Bile duct obstruction, extrahepatic; Stricture; Calculus; Extracorporeal shockwave lithotripsy

\section{INTRODUCTION}

Chronic pancreatitis is a chronic fibro-inflammatory disorder of the pancreas due to varying causes that leads to worsening of both exocrine and endocrine derangements as the disease progresses. ${ }^{1}$ Along with functional deterioration of the pancreas, morphologic changes including calcification, ductal ectasia, and parenchymal atrophy become more evident as the disease advances towards the endstage. ${ }^{1}$ Among these structural alterations, calcification can form in the pancreatic parenchyma and/or within the ductal lumen. The presence of calcification itself does not always elicit or correlate with symptom development such as pain. However, if the pain is considered to be caused by obstructing pancreatic calculi that induces pancreatic ductal hypertension with resultant increase in parenchymal pressure, decompression of the pancreatic duct is required. ${ }^{2}$ Ductal decompression can be done either endoscopically or surgically. Although surgical drainage is effective and more durable than endoscopic treatment, operative man- 
agement entails higher morbidity and mortality, thereby making endoscopic therapy a good alternative as first-line therapeutic modality. ${ }^{3}$ Since the majority of chronic pancreatitis patients with obstructing pancreatic duct stones have coexisting pancreatic duct stricture that renders stone extraction practically impossible without initial fragmentation, extracorporeal shock wave lithotripsy (ESWL), intraductal laser lithotripsy or electrohydraulic lithotripsy are employed to break up the stones prior to their removal, ${ }^{2-4}$ even when accompanied by pseudocysts. ${ }^{5}$

Along with the aforementioned morphologic changes, another structural alteration that can be seen in chronic pancreatitis patients is biliary stricture. Biliary stricture is not uncommon and has been reported to be incidentally found in up to $17 \%$ of patients with chronic pancreatitis. ${ }^{6}$ Although most are just anatomical narrowings that do not cause obstructive symptoms and many are imaging phenomenon alone, treatment is required when the patient develops obstructive symptoms. ${ }^{7,8}$ Chronic pancreatitis with obstructing stones which require ESWL to facilitate endoscopic stone extraction include patients with the most severe form of chronic pancreatitis. However, how often biliary stricture in this subset of patients is clinically significant has yet to be defined. The aim of this study was to assess the significance of biliary stricture in patients undergoing ESWL to remove obstructing pancreatic duct stones.

\section{MATERIALS AND METHODS}

\section{Patients and definitions}

A total of 97 chronic pancreatitis patients with abdominal pain due to obstructing pancreatic duct stones underwent ESWL followed by endoscopic retrograde cholangiopancreatography (ERCP) to remove obstructing stones from October 2014 to October 2017 at Virginia Mason Medical Center. Data on patient characteristics, the most likely cause of chronic pancreatitis, presence of biliary stricture and pseudocyst, laboratory results, and surgical management were collected by retrospectively reviewing the electronic medical records. This study was approved by the Institutional Review Board of our hospital (IRB number: IRB17-132).

Bile duct dilation was defined as bile duct diameter of $>10 \mathrm{~mm}$. Pancreatic duct dilation was considered to be present if the duct diameter was increased to $>6 \mathrm{~mm}$. Significant biliary stricture (SBS) was defined as biliary stricture with upstream bile duct dilatation $(>10 \mathrm{~mm})$ identified on abdominal imaging study that was accompanied by cholangitis, cholestatic liver enzyme elevation or symptomatic stricture with comparable complications requiring stent insertion at an outside hospital.

\section{Procedure and outcomes}

Computed tomography and/or magnetic resonance imaging/magnetic resonance cholangiopancreatography were obtained in all patients prior to ESWL to evaluate for biliary and pancreatic ductal changes, concomitant malignancy, presence of pseudocyst, and severity and localization of pancreatic calcification. ESWL and ERCP were performed in the same endoscopy procedure suite. Initially, the patient was placed in supine position and ESWL was performed using Storz Modulith ${ }^{\circledR}$ SLX-F2 lithotripter (Storz-Medical, Kreuzlingen, Switzerland) under general anesthesia. Pancreatic duct stones were exposed to 3,000 to 5,500 shock waves at power level 6 or 7 and a shock wave rate set to match the heart rate of the patient. If the stone showed no signs of being fragmented, the power level was increased until there was evidence of stone breakage. Immediately after ESWL, the patient was moved to the fluoroscopy table in the same room and underwent ERCP for stone extraction. Shape and length of biliary stricture, and the diameter of dilated upstream duct was definitively measured and evaluated at the time of ERCP using fluoroscopic images. When SBS was present, either a plastic prosthesis (Fig. 1) or fully covered self-expandable stent (fcSEMS) (Fig. 2) was inserted. The choice of the stent was left at the discretion of the endoscopist. For fcSEMS, Viabil stents (W.L. Gore \& Associates, Flagstaff, AZ, USA) and Wallflex biliary RX fully covered stent (Boston Scientific, Natick, MA, USA) were used with diameter ranging from $8 \mathrm{~mm}$ to $10 \mathrm{~mm}$ depending on the anatomy of stricture. As for plastic prosthesis, one or more 10-F plastic stent was inserted. Balloon dilatation of the stricture was performed if deemed necessary by the physician. Repeat ERCP was scheduled after 3 months when plastic stent was inserted and after 6 months when fcSEMS was placed. ERCP was performed earlier if obstructive symptoms developed. If the stricture resolved after this time period, biliary prosthesis was removed. However, if stricture persisted after plastic stent removal, it was replaced by either fcSEMS or multiple plastic stents. If fcSEMS was previously inserted, it was exchanged for another fcSEMS.

The primary outcomes were incidence of SBS and its associated risk factors. Secondary outcomes were success rate of SBS resolution, SBS recurrence rate, and the need for operative management. Success was defined as increase in $>75 \%$ of stricture diameter measured by cholangiogram at the time of stent insertion and removal. If stent was inserted for more than 1 year, it was considered a failure. Recurrence was defined as redevelopment of SBS after initial successful stricture resolution as defined above.

\section{Statistical analysis}

Statistical analyses were performed using IBM SPSS Sta- 

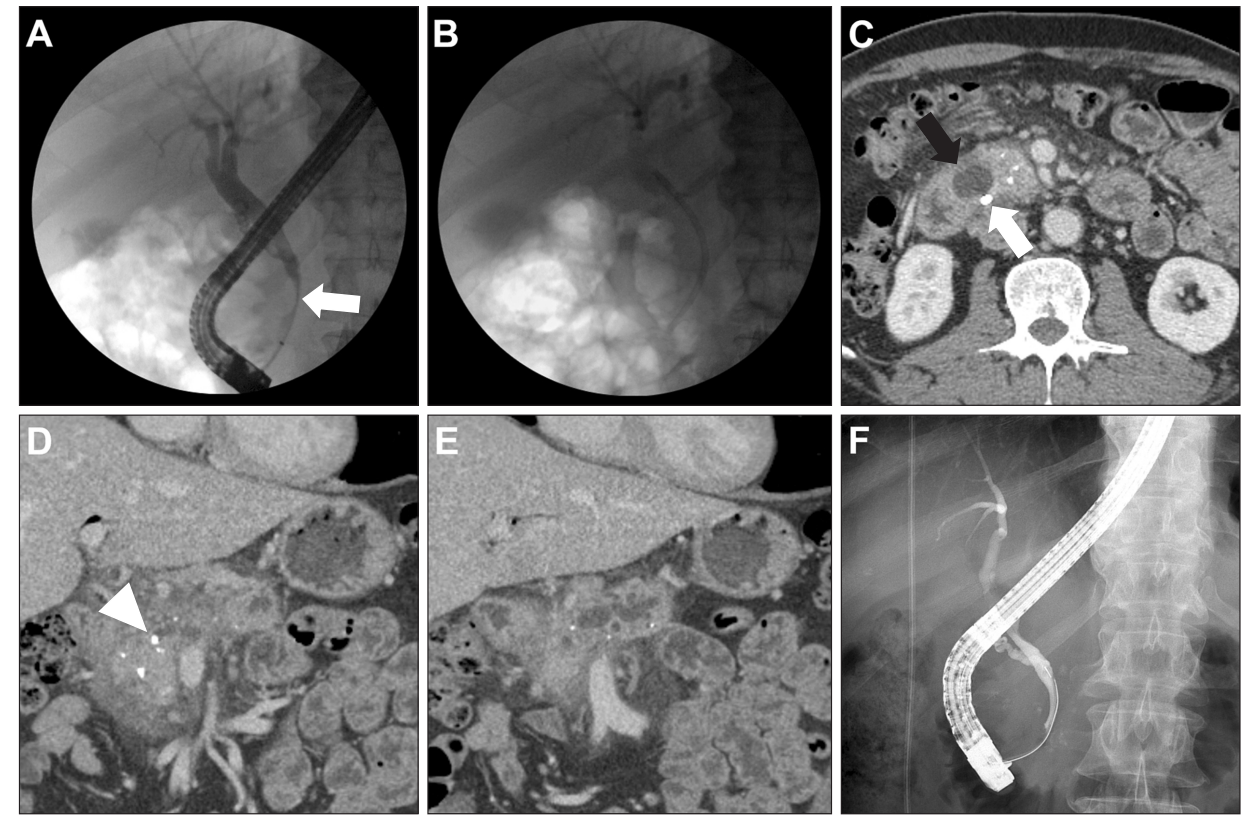

Fig. 1. Plastic stent insertion for an intrapancreatic common bile duct (CBD) stricture. (A) Endoscopic retrograde cholangiopancreatography performed at an outside hospital showed a biliary stricture at the distal CBD (white arrow) with upstream dilatation. (B) A plastic stent was inserted. (C) A follow-up computed tomography scan performed before extracorporeal shock wave lithotripsy (ESWL) showed a pseudocyst at the pancreatic head (black arrow) contiguous with the previously inserted plastic stent (white arrow). (D) On the coronal scan, an obstructing pancreatic duct stone (arrowhead) can be seen. (E) Pancreatic edema and peripancreatic infiltration, along with upstream duct dilatation, can also be observed. (F) A cholangiogram acquired 3 months after ESWL showed resolution of the biliary stricture along with the decompressed bile duct.
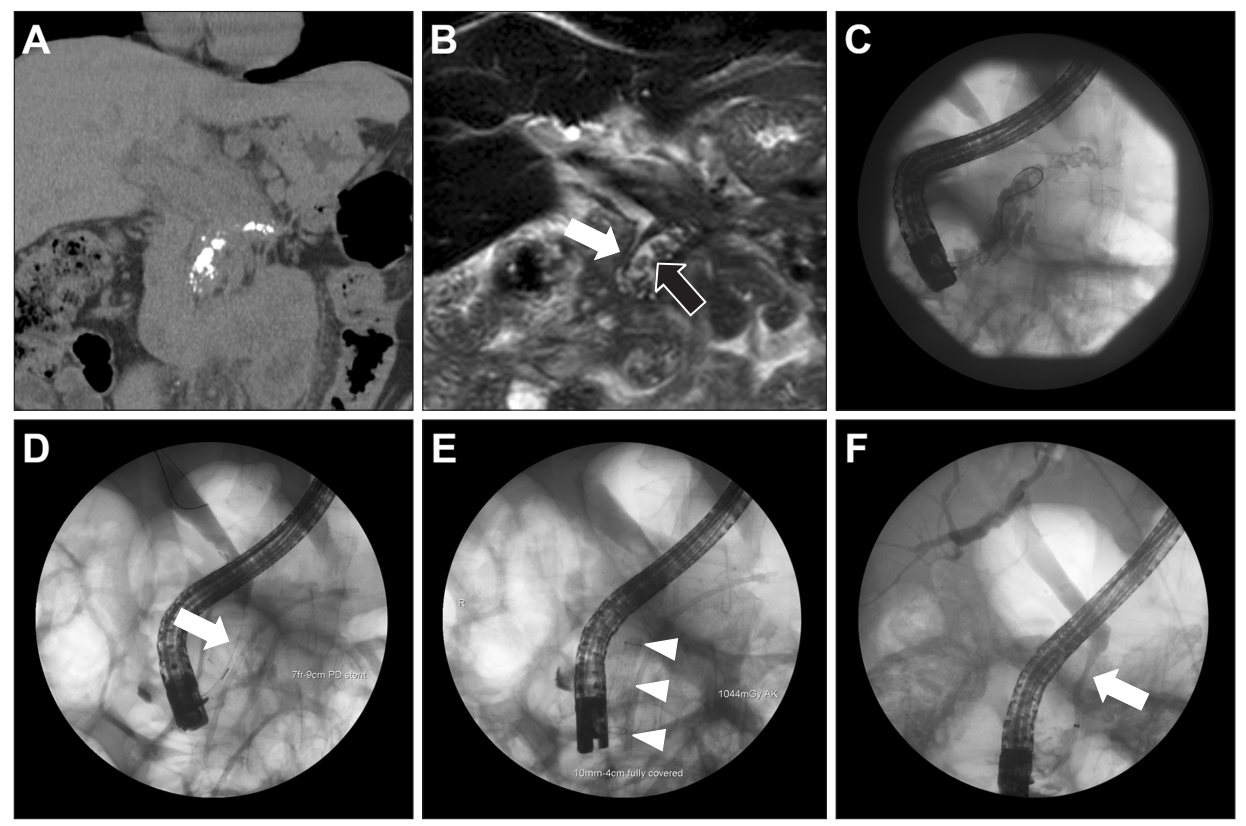

Fig. 2. Self-expandable stent (SEMS) insertion for an intrapancreatic common bile duct stricture. (A) An abdominal computed tomography scan performed at an outside hospital showed numerous calcific stones filling the entire pancreatic duct. (B) Magnetic resonance imaging performed before endoscopic retrograde cholangiopancreatography (ERCP) demonstrated a tapered narrowing of the intrapancreatic portion of the bile duct (white arrow) that appeared externally compressed by the dilated pancreatic duct filled with multiple filling defects (open arrow). (C) Many calcified stones were removed from the dilated pancreatic duct during ERCP after extracorporeal shock wave lithotripsy. (D) A tapered stricture is evident on the cholangiogram (white arrow). (E) A 1 $\times 4 \mathrm{~cm}$ fully covered SEMS (arrowheads) was inserted across the stricture. (F) A follow-up ERCP after 6 months showed improvement in the biliary stricture (white arrow). 
tistics version 23.0 (IBM Corp., Armonk, NY, USA). Data are expressed as mean \pm standard deviation or number (\%) values. Continuous and categorical variables were compared using the Mann-Whitney $U$ test and Fisher exact test, respectively. A two-sided p-values $<0.05$ were considered statistically significant.

\section{RESULTS}

\section{Clinical characteristics}

Approximately half of the patients in each group were male (Table 1). The average age of patients in the SBS group and the non-SBS group was 54 years and 51 years, respectively $(\mathrm{p}=0.52)$. There were also no differences regarding the prevalence of diabetes and smoking status between the groups. The most common cause of chronic pancreatitis was alcohol followed by idiopathic pancreatitis in both groups.

\section{Primary outcomes}

Biliary stricture was present in 35\% (34/97) of patients, and SBS was found in 11\% (11/97) at the time of ESWL

Table 1. Comparison of Clinical Characteristics and Outcomes According to the Significance of Biliary Stricture

\begin{tabular}{|c|c|c|c|}
\hline Variable & $\begin{array}{l}\text { SBS group } \\
(n=11)\end{array}$ & $\begin{array}{c}\text { Non-SBS group } \\
(n=86)\end{array}$ & $\mathrm{p}$-value \\
\hline Male sex & 6 (55) & 46 (54) & 0.97 \\
\hline Age, yr & $54.3 \pm 16.6$ & $51.2 \pm 14.6$ & 0.52 \\
\hline Weight, kg & $67.3 \pm 20.2$ & $72.8 \pm 18.9$ & 0.41 \\
\hline Diabetes mellitus & $6(54)$ & $36(42)$ & 0.52 \\
\hline Smoking & & & 0.17 \\
\hline Never & $1(9)$ & $28(33)$ & \\
\hline Former/current & $10(91)$ & $58(67)$ & \\
\hline Chronic pancreatitis cause & & & 0.26 \\
\hline Alcohol & $8(73)$ & $42(49)$ & \\
\hline Idiopathic & $2(18)$ & $31(36)$ & \\
\hline Familial (hereditary) & 0 & $3(4)$ & \\
\hline Tropical & 0 & $5(6)$ & \\
\hline Autoimmune & $1(9)$ & $1(1)$ & \\
\hline Pancreas divisum & 0 & $4(5)$ & \\
\hline Biliary stricture & $11(100)$ & $23(27)$ & $<0.01$ \\
\hline Pseudocyst & $4(36)$ & 7 (8) & 0.02 \\
\hline Duodenal groove & 0 & 1 & \\
\hline Pancreas head & 4 & 0 & \\
\hline Pancreas body & 0 & 5 & \\
\hline Pancreas tail & 0 & 1 & \\
\hline Surgery & $1(9)$ & $4(5)$ & 0.46 \\
\hline Whipple/PPPD & 0 & 2 & \\
\hline Distal pancreatectomy & 0 & 2 & \\
\hline Frey procedure & 1 & 0 & \\
\hline
\end{tabular}

Data are presented as number (\%) or mean \pm SD.

SBS, significant biliary stricture; PPPD, pylorus-preserving pancreaticoduodenectomy.
(Table 1). Pseudocyst was present in 36\% (4/11) of patients with SBS compared to $8 \%(7 / 86)$ of those without SBS $(\mathrm{p}=0.02)$. All pseudocysts were located in the pancreas head $(n=4)$ in the SBS group. Pseudocyst in 27\% (3/11) of SBS patients were contiguous with the bile duct and were thought to be externally compressing the bile duct, and pseudocyst was considered to be contributing to biliary obstruction. In contrast, pseudocysts in the non-SBS group were not located in the vicinity of the bile duct but in the duodenal groove $(n=1)$, body $(n=4)$, and body/tail $(n=1)$.

\section{Secondary outcomes}

SBS was initially managed by insertion of either a plastic prosthesis $(n=7)$ or fcSEMS $(n=4)$ (Table 2). Of those with SBS, four patients had stent inserted at outside hospital with two patients having plastic stent inserted and the other two patients fcSEMS placed prior to being transferred. Initial success rate of fcSEMS in stricture resolution was $75 \%$ (3/4) since one patient required more than 1 year of stent insertion. Initial success rate of a single plastic stent was $29 \%(2 / 7)$. In the remaining five patients, single plastic stent was changed to either fcSEMS $(n=4)$ or multiple plastic stents $(n=1)$. After changing to fcSEMS or multiple plastic stents, $60 \%(3 / 5)$ showed stricture resolution.

Table 2. Details on SBS Patients Undergoing Stent Placement for Biliary Stricture

\begin{tabular}{|c|c|}
\hline Variable & Value \\
\hline \multicolumn{2}{|l|}{ Initial stenting ( $n=11$ ) } \\
\hline \multicolumn{2}{|l|}{ Stent type } \\
\hline fcSEMS & $4(36)$ \\
\hline Single plastic stent & $7(64)$ \\
\hline \multicolumn{2}{|l|}{ Stricture resolution } \\
\hline fcSEMS & $3 / 4(75)$ \\
\hline Single plastic stent & $2 / 7(29)$ \\
\hline \multicolumn{2}{|l|}{ Stent insertion duration, wk } \\
\hline fcSEMS & $19.4 \pm 5.8$ \\
\hline Single plastic stent & $11.9 \pm 2.6$ \\
\hline \multicolumn{2}{|l|}{ Stenting after failure with a single plastic stent $(n=5)$} \\
\hline \multicolumn{2}{|l|}{ Stent type } \\
\hline fcSEMS & $4(80)$ \\
\hline Multiple plastic stents & $1(20)$ \\
\hline Stricture resolution & $3(60)$ \\
\hline Stent insertion duration, wk & $55.1 \pm 56.1$ \\
\hline \multicolumn{2}{|l|}{ Final outcomes $(n=11)$} \\
\hline Overall follow-up duration, wk & $98.8 \pm 70.3$ \\
\hline Overall stricture resolution & 8 (73) \\
\hline Follow-up duration after initial stricture resolution, wk $(n=8)$ & $72.3 \pm 78.9$ \\
\hline No recurrence & $6 / 8(75)$ \\
\hline Recurrence & $2 / 8(25)$ \\
\hline Time to recurrence, yr & $3,3.5$ \\
\hline Stricture resolution failure & $3(27)$ \\
\hline
\end{tabular}

Data are presented as number (\%) or mean \pm SD.

SBS, significant biliary stricture; fcSEMS, fully covered self-expandable metallic stent. 
Therefore, overall success rate was $73 \%$ (8/11). Overall follow-up duration from stent insertion to last follow-up was $98.8 \pm 70.3$ weeks. As for the eight patients who were successful in SBS resolution, they have been followed-up for $72.3 \pm 78.9$ weeks after initial SBS resolution. Among these patients, two patients showed recurrence after 3 years and 3.5 years each. In this series of patients with severe chronic pancreatitis, one patient in SBS group and four patients in non-SBS group ultimately required surgery (Table 1). The reasons for surgical intervention were for refractory pain along with recurrent stricture in one patient with SBS and for refractory pain $(\mathrm{n}=3)$ or disconnected duct syndrome $(\mathrm{n}=1)$ in patients without SBS. Two patients underwent Whipple/PPPD (pylorus-preserving pancreaticoduodenectomy), two received distal pancreatectomy with splenectomy, and one had a Frey procedure.

\section{DISCUSSION}

The incidence of intrapancreatic common bile duct (CBD) stricture has been reported to vary widely from $3 \%$ to $46 \%$ depending on the definition of stricture, patient population, and diagnostic modality. ${ }^{9}$ In the present study, biliary stenoses were noted in approximately one third $(35 \%, 34 / 97)$ of the subjects which falls into the upper range of previously reported incidence. This is conceivable since our study population, chronic pancreatitis patients with obstructing pancreatic duct stones requiring ESWL, represent the most severe form of chronic pancreatitis. Among these aforementioned patients, approximately one third $(32 \%, 11 / 34)$ had SBS and one third of the latter $(36 \%$, 4/11) had a concomitant pseudocyst.

Since pseudocysts were found more frequently in patients with SBS compared to those without SBS (36\% vs $8 \%, p=0.02$ ), pseudocyst may be a factor related to the development of SBS. Considering that all pseudocysts were located in pancreas head, 75\% (3/4) of which were contiguous with the bile duct, pseudocyst itself could be regarded as a pertinent element in SBS development by extrinsically compressing the intrapancreatic CBD (Fig. 1). ${ }^{10}$ Alternatively, pseudocyst may not be the cause of SBS but a byproduct that reflects the presence of acute inflammation. Acute inflammation of the pancreas causes parenchymal edema and when it involves the pancreas head, can result in extrinsic compression of intrapancreatic CBD which could exacerbate SBS formation. ${ }^{9}$ Whether pseudocyst is the cause of SBS or simply a byproduct of the inflammatory process, presence and location of pseudocyst seems to have significance at least during acute symptomatic exacerbations of chronic pancreatitis. However, the observation that two thirds $(64 \%, 7 / 11)$ of SBS patients did not have a pseudocyst signifies that pseudocyst by itself is usually not the sole determinant factor, albeit one of the important contributing factors to SBS formation. ${ }^{11-13}$ The principal mechanism behind biliary stricture formation in the vast majority of chronic pancreatitis patients still remains one of progressive fibrosis of the pancreatic parenchyma due to recurrent acute/chronic inflammation resulting in a fibrotic stricture of intrapancreatic $\mathrm{CBD} .{ }^{1,9,13}$ In the present study, those who recurred did not have pseudocyst at diagnosis and there was no pseudocyst at the time of recurrence. Recurrence was deemed to be due to fibrosis and calcification.

Presence of intrapancreatic CBD stricture per se is not an indication for therapy, but if patients also have clinical evidence of biliary obstruction (cholangitis, jaundice, or cholestatic liver enzyme elevations), biliary drainage is recommended. ${ }^{7,8}$ Surgical bypass has historically been the definitive treatment for managing intrapancreatic CBD strictures. However, endotherapy is often chosen as firstline therapeutic modality because it is less invasive and has a lower risk of morbidity and mortality. Moreover, at times, it can be used as a bridge to surgery, sometimes because of comorbid conditions of the patient, and at other times due to patients' preference or refusal to undergo operation. When physician opts for endoscopic biliary stent placement, either plastic or fcSEMS can be used. Stricture resolution rates at fcSEMS removal have been reported to range from $37 \%$ to $95 \%$ with median time to SEMS removal being 5.5 to 7.5 months. ${ }^{7,14-20}$ Similar to fcSEMS, stricture resolution rates of multiple plastic stents range from $44 \%$ to $92 \%,{ }^{21-23}$ although placement of plastic endoprosthesis has required longer stenting duration (mean, 14 to 21 months) to achieve stricture resolution, most likely due to the necessity of frequent reinterventions and gradual stricture dilation by increasing the number of stents. In line with these findings, the stricture resolution rate of fCSEMS in the current study was $75 \%$ when it was chosen initially, and that of fCSEMS and multiple plastic stents was $60 \%$ when they were inserted following failed stricture resolution using an initial single plastic stent. When a single 10-F plastic stent was initially inserted, our stricture resolution rate was only $29 \%$ which is within the lower range of previously reported resolution rates ( $12 \%$ to $80 \%$ ), although these data are derived from reports of long-term success rates with mean stenting duration of 9 to 21 months. ${ }^{9} \mathrm{Di}$ rect comparison between fcSEMS and plastic stents is not feasible in the present study because if the stricture did not resolve at 3-month follow-up ERCP after a single plastic stent insertion, it was changed to either fcSEMS or multiple plastic stents. Nevertheless, it is worth noting that the 
stricture responded to the placement of a single $10-\mathrm{F}$ plastic stent in two patients, one of whom had a pseudocyst contiguous with the stricture (Fig. 1).

The findings that a single plastic stent for 3 months was sufficient for stricture resolution in two patients and that the resolution rate of stricture was encouraging with fcSEMS or multiple plastic stents imply that acute inflammation played an important role in SBS formation. How could patients with the most severe form of chronic pancreatitis known to be resistant to stricture dilation have shown a favorable response to endoscopic stent insertion? One reason for the high success rate may be that patients included in the current study had abdominal pain induced by obstructing pancreatic duct stones that required ESWL. Removal and clearance of pancreatic duct stones, in turn decompresses the elevated pancreatic ductal pressure, which in turn would have led not only to mitigation of pain but also amelioration of acute inflammation and edema aiding in resolution of SBS.

Interestingly, the main reason for operation in our patient series was not for stricture recurrence or progression of biliary stricture, but rather for refractory pain. Of the five patients who underwent surgery, only one patient was from SBS group and the reason was mainly for refractory pancreatic-type pain although it was also accompanied by recurrent stricture, which resolved after the operation. The indication for surgery was also for ongoing pain in the patients without SBS. Although extensive evaluation had been done to substantiate that the cause of pain was obstructing pancreatic duct stone prior to ESWL, finding that four patients underwent operation for ongoing and refractory pain after clearance of pancreatic duct stones suggests that the cause of abdominal pain was not solely due to elevated ductal pressure from obstructing pancreatic calculi but potentially to persistent inflammation, capsule distension, or perineural fibrosis. ${ }^{2}$

There are several limitations to this study. First, followup period was not long enough to adequately capture the long-term recurrence of SBS strictures or the subsequent development of malignancy. A previous report has shown that recurrence rate of biliary stricture due to chronic pancreatitis can be as high as $40.6 \% .{ }^{24}$ Since there was recurrence of SBS in two patients, 3 years and 3.5 years following initial successful stricture resolution in the current series, it is likely that additional cases of SBS recurrence might be encountered with prolonged follow-up. In the latter setting, one needs to be aware of the risk of malignancy development in chronic pancreatitis and one cannot simply assume that this is a benign stricture. Second, the retrospective nature of the study along with many patients being referred from outside hospitals could have led to selection bias and also resulted in limited availability of pertinent laboratory findings such as carbohydrate antigen 19-9, amylase/lipase, and sequential liver function test prior to the development of SBS. Presence of jaundice and/or alkaline phosphatase elevation for more than 1 month has been shown to increase the development of biliary cirrhosis but not all laboratory results prior to being transferred were available. Third, relatively small number of patients with SBS with resultant small number patients undergoing endoscopic management along with mixed use of plastic stent and metal stent limited assessment of variables to define potential statistical significance between the two groups of patients. Lack of uniform strategy in placing subsequent stent(s) in case of failed resolution of stricture with first stent is another limitation of this study. As such, a randomized multicenter study with larger number of patients may be warranted in the future.

In conclusion, progressive periductal fibrosis due to chronic inflammation seems to be the basic underlying pathogenesis of biliary stricture development in patients with chronic pancreatitis. However, obstructing pancreatic calculi that lead to inflammation and edema may also be associated with ductal rupture and pseudocyst. The latter seems to be an important component to the development of SBS during the acute phase. Although combination of ESWL and pancreatic stone removal plus endoscopic stenting showed encouraging stricture resolution rates, we should be cognizant of the fact that chronic pancreatitis is a progressive disease and as such, careful long-term follow-up of these patients is mandatory despite initial successful treatment.

\section{CONFLICTS OF INTEREST}

No potential conflict of interest relevant to this article was reported.

\section{ACKNOWLEDGEMENTS}

The authors thank Terri Davis Smith, Academic Specialist (Digestive Disease Institute, Virginia Mason Medical Center, Seattle, WA, USA) for administrative, technical, and material support.

\section{AUTHOR CONTRIBUTIONS}

Study conception and design: R.A.K. Data acquisition: J.J.H. Data analysis and interpretation: J.J.H., R.A.K., M.G. Drafting of the article: J.J.H., R.A.K. Critical revision of 
the article for important intellectual content: S.S.I., A.S.R., M.C.L., M.G., R.A.K. Statistical analysis: J.J.H. Study supervision: R.A.K. Final approval of the article: all authors.

\section{ORCID}

Jong Jin Hyun https://orcid.org/0000-0002-5632-7091

Shayan S. Irani https://orcid.org/0000-0002-5490-692X

Andrew S. Ross https://orcid.org/0000-0002-6587-7503

Michael C. Larsen https://orcid.org/0000-0003-2154-7876

Michael Gluck https://orcid.org/0000-0002-2048-3949

Richard A. Kozarek

https://orcid.org/0000-0002-9745-7538

\section{REFERENCES}

1. Kleeff J, Whitcomb DC, Shimosegawa T, et al. Chronic pancreatitis. Nat Rev Dis Primers 2017;3:17060.

2. Drewes AM, Bouwense SAW, Campbell CM, et al. Guidelines for the understanding and management of pain in chronic pancreatitis. Pancreatology 2017;17:720-731.

3. Seven G, Schreiner MA, Ross AS, et al. Long-term outcomes associated with pancreatic extracorporeal shock wave lithotripsy for chronic calcific pancreatitis. Gastrointest Endosc 2012;75:997-1004

4. Attwell AR, Patel S, Kahaleh M, Raijman IL, Yen R, Shah RJ. ERCP with per-oral pancreatoscopy-guided laser lithotripsy for calcific chronic pancreatitis: a multicenter U.S. experience. Gastrointest Endosc 2015;82:311-318.

5. Li BR, Liao Z, Du TT, et al. Extracorporeal shock wave lithotripsy is a safe and effective treatment for pancreatic stones coexisting with pancreatic pseudocysts. Gastrointest Endosc 2016;84:69-78.

6. Kalvaria I, Bornman PC, Marks IN, Girdwood AH, Bank L, Kottler RE. The spectrum and natural history of common bile duct stenosis in chronic alcohol-induced pancreatitis. Ann Surg 1989;210:608-613.

7. Irani S, Baron TH, Akbar A, et al. Endoscopic treatment of benign biliary strictures using covered self-expandable metal stents (CSEMS). Dig Dis Sci 2014;59:152-160.

8. Warshaw AL, Schapiro RH, Ferrucci JT Jr, Galdabini JJ. Persistent obstructive jaundice, cholangitis, and biliary cirrhosis due to common bile duct stenosis in chronic pancreatitis. Gastroenterology 1976;70:562-567.

9. Familiari P, Boškoski I, Bove V, Costamagna G. ERCP for biliary strictures associated with chronic pancreatitis. Gastrointest Endosc Clin N Am 2013;23:833-845.

10. Delhaye M, Arvanitakis M, Bali M, Matos C, Devière J. Endoscopic therapy for chronic pancreatitis. Scand J Surg 2005;94:143-153.
11. Gadacz TR, Lillemoe K, Zinner M, Merrill W. Common bile duct complications of pancreatitis evaluation and treatment. Surgery 1983;93:235-242.

12. Singh SM, Reber HA. The pathology of chronic pancreatitis. World J Surg 1990;14:2-10.

13. Warshaw AL, Rattner DW. Facts and fallacies of common bile duct obstruction by pancreatic pseudocysts. Ann Surg 1980;192:33-37.

14. Cahen DL, Rauws EA, Gouma DJ, Fockens P, Bruno MJ. Removable fully covered self-expandable metal stents in the treatment of common bile duct strictures due to chronic pancreatitis: a case series. Endoscopy 2008;40:697-700.

15. Perri V, Boškoski I, Tringali A, et al. Fully covered self-expandable metal stents in biliary strictures caused by chronic pancreatitis not responding to plastic stenting: a prospective study with 2 years of follow-up. Gastrointest Endosc 2012;75:1271-1277.

16. Kahaleh M, Brijbassie A, Sethi A, et al. Multicenter trial evaluating the use of covered self-expanding metal stents in benign biliary strictures: time to revisit our therapeutic options? J Clin Gastroenterol 2013;47:695-699.

17. Devière J, Nageshwar Reddy D, Püspök A, et al. Successful management of benign biliary strictures with fully covered selfexpanding metal stents. Gastroenterology 2014;147:385-395.

18. Saxena P, Diehl DL, Kumbhari V, et al. A US multicenter study of safety and efficacy of fully covered self-expandable metallic stents in benign extrahepatic biliary strictures. Dig Dis Sci 2015;60:3442-3448.

19. Coté GA, Slivka A, Tarnasky P, et al. Effect of covered metallic stents compared with plastic stents on benign biliary stricture resolution: a randomized clinical trial. JAMA 2016;315:1250-1257.

20. Chaput U, Vienne A, Audureau E, et al. Temporary placement of fully covered self-expandable metal stents for the treatment of benign biliary strictures. United European Gastroenterol J 2016;4:403-412.

21. Draganov P, Hoffman B, Marsh W, Cotton P, Cunningham J. Long-term outcome in patients with benign biliary strictures treated endoscopically with multiple stents. Gastrointest Endosc 2002;55:680-686.

22. Catalano MF, Linder JD, George S, Alcocer E, Geenen JE. Treatment of symptomatic distal common bile duct stenosis secondary to chronic pancreatitis: comparison of single vs. multiple simultaneous stents. Gastrointest Endosc 2004;60:945-952.

23. Pozsár J, Sahin P, László F, Forró G, Topa L. Medium-term results of endoscopic treatment of common bile duct strictures in chronic calcifying pancreatitis with increasing numbers of stents. J Clin Gastroenterol 2004;38:118-123.

24. Park JS, Lee SS, Song TJ, et al. Long-term outcomes of covered self-expandable metal stents for treating benign biliary strictures. Endoscopy 2016;48:440-447. 